Original research article

\title{
The effect of different training modalities on resting hormonal level in active young males
}

\author{
Matej Vajda ${ }^{1 *}$, Marián Vanderka ${ }^{2}$, Gabriel Buzgó ${ }^{2}$, Milan Sedliak ${ }^{2}$, Tomáš Kampmiller ${ }^{2}$ \\ ${ }^{1}$ Comenius University in Bratislava, Faculty of Physical Education and Sports, Hamar Institute for Human Performance, Bratislava, Slovak Republic \\ ${ }^{2}$ Comenius University in Bratislava, Faculty of Physical Education and Sports, Bratislava, Slovak Republic
}

\begin{abstract}
The purpose of this study was to determine the changes in the resting level of serum cortisol, testosterone and $\mathrm{T} / \mathrm{C}$ ratio in response to different training modalities and their variations. A secondary purpose was to identify if the various six weeks training programs are an effective way to improve physical fitness. 86 regularly active young males were assigned to one of six groups: Endurance constant running (ECR), Endurance interval running (EIR), Resistance training (RT), Explosive training (ET), Speed-endurance $50 \mathrm{~m}$ running (SER50) and Speed-endurance $150 \mathrm{~m}$ running (SER150) training. The resting levels of testosterone, cortisol and $\mathrm{T} / \mathrm{C}$ ratio, as well as physical fitness, were measured. The ECR, EIR, and RT training program decreased COR level $(P<0.05)$. An increase of the T/C ratio was observed in the ECR and EIR group $(P<0.05)$. Except for SER50, each training program improved physical fitness. Our results suggest that endurance and resistance training modalities performed with a moderate to vigorous intensity may be a usable way to manage the resting cortisol level and enhance physical fitness in active young males.
\end{abstract}

Keywords: Cortisol; Endurance training; Resistance training; Resting hormonal level

Highlights:

- The endurance and resistance training positively affected the endocrine system by reducing the resting level of cortisol.

- The testosterone remained unchanged following any training programs and their variation.

- Short-term duration training programs (6 weeks) can enhance the physical fitness of active young males.

\section{Introduction}

The training program is planned, structured and repetitive in the sense of improvement or maintenance of one or more components of physical fitness (Caspersen et al., 1985). As has been presented earlier, adaptive changes in physical fitness are highly specific to the training programs involved (Kraemer et al., 1996). In general, strength training increases muscle strength, power, as well as muscle hypertrophy (Häkkinen et al., 2003), while endurance training leads to gains in endurance parameters such as maximal oxygen consumption, maximal aerobic power output and endurance performance tests (Grandys et al., 2009). However, the effects on nonspecific components are limited. Besides these facts, the crucial variables of any training programs are also the volume, intensity, and frequency of training sessions. The combination of these training variables determines the magnitude of adaptive responses that either enhance, maintain or decrease physical fitness (Hawley, 2008).

In addition to these changes in physical fitness, physical activity also produces specific variations in the concentration of endogenous hormones. The exercise-associated hormonal changes, mainly in circulating level of cortisol (COR), testosterone (TST) and their relationship presented by testosterone/ cortisol ratio (T/CR) (Doma et al., 2015; Hough et al., 2013; Kilian et al., 2016; Popovic et al., 2019) have been extensively studied. Attention has previously been directed towards acute hormonal response to a single session of exercise (Popovic et al., 2019), persistent changes in the acute response to an exercise stimulus (Walker et al., 2017), as well as chronic changes in resting hormone concentrations after a training program (Alghadir et al., 2015; Shakeri et al., 2012). The magnitude of acute and chronic variations of hormones depends on the training variables, such as type of the training modalities (Schumann et al., 2015), duration of the training program (Shakeri et al., 2012), as well as intensity and volume of the training sessions (Kilian et al., 2016; Peake et al., 2014). Also, the subjects' experience (Popovic et al., 2019), gender and age category (Häkkinen et al., 2000) are essential factors affecting hormonal response to exercise and training. Currently, few and conflicting data are available about the resting hormonal responses to different training modalities. The data is mainly focused on strength training and less on endurance and

\footnotetext{
* Corresponding author: Matej Vajda, Comenius University in Bratislava, Faculty of Physical Education and Sports, Hamar Institute for Human Performance, Nabr. L. Svobodu 9, 81469 Bratislava, Slovak Republic; e-mail: matej.vajda@uniba.sk http://doi.org/10.32725/jab.2021.008

Submitted: 2020-05-11 • Accepted: 2021-03-08 • Prepublished online: 2021-03-09

J Appl Biomed 19/2: 83-90 • EISSN 1214-0287 • ISSN 1214-021X

(c) 2021 The Authors. Published by University of South Bohemia in České Budějovice, Faculty of Health and Social Sciences.

This is an open access article under the CC BY-NC-ND license.
} 
speed-endurance training in active young males (Ahtiainen et al., 2003; Alghadir et al., 2015; Grandys et al., 2009; Kraemer et al., 1999; Shakeri et al., 2012). However, to our knowledge, there are not yet any studies that would compare the effects of these different training modalities (endurance, strength, and speed-endurance) in combination with different exercise intensities on the resting hormonal level and physical fitness within a short-term duration among active young males.

To optimize the training activity prescription for active young males, it appears reasonable to identify the effective training modalities with the combination of intensity and volume of the training programs to promote both physical fitness and endocrine health benefits. Therefore, the present study aimed to determine the response of the resting level of serum cortisol, testosterone and testosterone/cortisol ratio to different training modalities and their variations in active young males. A secondary purpose was to identify if the different training programs are an effective way to improve physical fitness according to the type of training modalities performed during six-week training programs.

\section{Materials and methods}

\section{Experimental design}

A six-groups longitudinal (pre-training, post-training) design was used. Two weeks before the enrolment in the training programs (before the first training), all subjects underwent a familiarization session (to familiarize them with the testing procedures) to minimize the learning effect during the study. Initial anthropometric and physical performance assessments were realized (3-5 days before the first training) by experienced examiners. Each training group underwent a specific training program; these are described below in the "Training programs" section. Each training program had the same sixweek duration (three sessions per week on non-consecutive days), and was performed under the supervision of certified strength and conditioning coaches.

Resting hormonal level was assessed at week 1 (morning of the first training session day) and week 6 (morning of the eighteenth training session day). Fasting (10 h) blood samples $(20 \mathrm{ml})$ at rested state were obtained on the training day (7:00-7:30) followed by the same standardized breakfast for all training protocols. Three to five days after the last training session, post-training physical fitness assessments were conducted. Each subject had their training and physical testing times standardized throughout the study to minimize the effects of diurnal variations.

\section{Subjects}

Initially, 86 students of a strength and conditional coaching program who regularly trained ( $3 \leq$ sessions per week of $60 \mathrm{~min}$ in last five years) in various physical activities volunteered to participate in this study. They were assigned to one of six groups: Endurance constant running (ECR), Endurance interval running (EIR), Resistance training (RT), Explosive training (ET), Speed-endurance $50 \mathrm{~m}$ running (SER50) and Speed-endurance $150 \mathrm{~m}$ running (SER150). All subjects were healthy and free of endocrine, cardiovascular and neuromuscular diseases - or any other diseases that may have affected the ability to perform the training and testing. Each subject claimed to be free of anabolic steroids or any other legal or illegal agents.

Two days before the beginning (pre-training physical fitness testing) of the study and throughout the study period, subjects were instructed to maintain their usual eating habits, normal daily activities, not to consume any medications or dietary supplements, and to refrain from other physical activities. Additionally, subjects were instructed to monitor their eating habits (time, type and amount of food consumed) in the 48 hours before the initial hormonal testing - and to replicate this during the second hormonal testing. Seven subjects did not complete the study, four subjects in the SER50 group as a result of injury, and one subject in the SER50, SER150 and EIR group due to an attendance of less than $90 \%$ at the training sessions. The final sample for statistical analyses included 79 subjects (Table 1 ).

Table 1. Sample characteristics before the beginning of the training program (mean $\pm S D$ )

\begin{tabular}{llll}
\hline & Age (years) & Height $(\mathrm{m})$ & Weight $(\mathrm{kg})$ \\
\hline $\operatorname{ECR}(n=15)$ & $22.34 \pm 0.57$ & $1.80 \pm 0.06$ & $77.43 \pm 7.46$ \\
$\operatorname{EIR}(n=13)$ & $22.96 \pm 1.14$ & $1.79 \pm 0.08$ & $76.28 \pm 7.11$ \\
$\operatorname{RT}(n=15)$ & $21.94 \pm 0.87$ & $1.82 \pm 0.05$ & $79.73 \pm 7.22$ \\
$\operatorname{ET}(n=17)$ & $22.56 \pm 1.13$ & $1.81 \pm 0.04$ & $80.21 \pm 8.25$ \\
$\operatorname{SER} 50(n=8)$ & $22.66 \pm 1.35$ & $1.78 \pm 0.05$ & $75.68 \pm 6.37$ \\
$\operatorname{SER} 150(n=11)$ & $22.63 \pm 1.88$ & $1.79 \pm 0.08$ & $76.76 \pm 7.77$ \\
\hline
\end{tabular}

The Ethical Committee of the Faculty of Physical Education and Sports, Comenius University in Bratislava, approved the study in accordance with the ethical standards of the Helsinki Declaration, and subjects were fully informed about the nature and possible risks of all procedures before providing written informed consent.

\section{Training programs}

Endurance training protocols

Training protocols were realized on an outdoor $350 \mathrm{~m}$ running track. Before each training and testing session, standardized warm-up protocol (jogging $700 \mathrm{~m}$ and running drills) with a total duration of 10-15 min was performed. This was identical for both groups. The intensity was established from the tempo of Cooper's 12-minute run test (TempoC). The tempo was calculated by a formula of distance covered/12 min. During the training, subjects received visual feedback of the running time and were encouraged verbally during the run.

The ECR group performed 30 mins continuous running with an intensity at $70 \%$ of TempoC. Intensity was expressed as running time per lap $(350 \mathrm{~m})$. The training volume was increased by 2 mins each week (from $30 \mathrm{~min}$ at week 1 to $40 \mathrm{~min}$ at week 6).

The EIR training protocol consisted of 10/10 interval running. This group performed 50 reps of $10 \mathrm{~s}$ intervals at intensity $115 \%$ of TempoC (with $10 \mathrm{~s}$ of passive resting periods in between). The intensity expressed the distance which should be covered during the $10 \mathrm{~s}$ run. This distance was marked by a stick with specific numbers for each subject. The training volume increased by three reps per week (from 50 reps at week 1 to 65 reps at week 6).

\section{Strength training protocols}

The standardized warm-up protocol was identical for both groups and was performed before each training and testing session. The warm-up protocol was a combination of dynamic and static stretching exercises, two sets $\times 10$ reps of push-ups and half-squat jumps with a total duration of 10-15 min. The volume progression was achieved by adding one set from the 
4th week onwards in both training programs. Also, progressive overload was applied by increasing the load when all prescribed repetitions for a particular exercise were achieved in two consecutive workouts.
The RT group performed traditional hypertrophic training consisting of 7 exercises with 90 seconds of rest periods between both sets and exercises. The characteristics of RT training are described in Table 2.

Table 2. Characteristics of the RT training program

\begin{tabular}{|c|c|c|c|c|c|}
\hline Exercise & $\begin{array}{c}\text { No. of sets } \\
\text { (in } 1-3 \text { week) }\end{array}$ & $\begin{array}{c}\text { No. of sets } \\
\text { (in } 4-6 \text { week) }\end{array}$ & Intensity & Reps & Tempo \\
\hline Deadlift & 3 & 4 & $8 \mathrm{RM}$ & 8 & 2121 \\
\hline Bench press & 3 & 4 & $8 \mathrm{RM}$ & 8 & 2021 \\
\hline Back squat & 3 & 4 & $8 \mathrm{RM}$ & 8 & 2021 \\
\hline Barbell row & 3 & 4 & $8 \mathrm{RM}$ & 8 & 2021 \\
\hline Pull-over & 3 & 4 & $10 \mathrm{RM}$ & 10 & 2020 \\
\hline Military press & 3 & 4 & $10 \mathrm{RM}$ & 10 & 2021 \\
\hline Biceps curl & 3 & 4 & $10 \mathrm{RM}$ & 10 & 2020 \\
\hline
\end{tabular}

The ET group carried out training focused on maximal acceleration during the concentric phase of the movement. The training contained seven exercises (Olympic lifts/velocity-dependent, ballistic and non-ballistic exercises), with a 90 sec- ond and 5 minutes rest period between the sets and exercises, respectively. During the ET, the subjects were encouraged to perform each lift as fast as possible. Table 3 shows the characteristics of ET training protocol.

Table 3. Characteristics of the ET training program

\begin{tabular}{lcclc}
\hline Exercise & $\begin{array}{c}\text { No. of sets } \\
\text { (in 1-3 week) }\end{array}$ & $\begin{array}{c}\text { No. of sets } \\
\text { (in 4-6 week) }\end{array}$ & Intensity & Reps \\
\hline Power clean & 2 & 3 & max. acceleration & 6 \\
Hang Power snatch & 2 & 3 & max. acceleration & 6 \\
Half squat jumps & 2 & 3 & max. acceleration & 8 \\
Bench pull & 2 & 3 & max. acceleration & 8 \\
Jerks & 2 & 3 & max. acceleration & 15 \\
Standing barbell twist & 2 & 3 & max. acceleration & 20 \\
Side step-side jump-vertical jump & 2 & 3 & max. acceleration & 10 \\
\hline
\end{tabular}

\section{Speed-endurance protocols}

Both speed-endurance training protocols consist of two specific training sessions ( $150 \mathrm{~m}$ or $50 \mathrm{~m}$ distance running), and one similar session (Tables 4 and 5 ) consists of 2 sets of 8 reps $\times 100 \mathrm{~m}$ running with a 1 and 5 min rest period between the reps and sets, respectively. Training programs were performed on an outdoor $350 \mathrm{~m}$ running track. Before each training and testing session, standardized warm-up protocol (jogging $700 \mathrm{~m}$, running drills and $3 \times 80 \mathrm{~m}$ incremental sprints) with a total duration of 10-15 min was performed - this was similar for both groups. The intensity of specific and similar sessions was predetermined from pre-testing assessment and was controlled in each training session throughout the study by dual-beam light timing gates (Vanel-gates, Vanel Ltd, Nizna, Slovakia). Subjects received visual feedback of their running time following each repetition and were encouraged verbally during the run.

The SER150 protocol included 1 set $\times 4$ reps of $150 \mathrm{~m}$ running (Table 4) with a 6 min rest period between the runs. The training volume progressed with the addition of one rep from the 4th week. The total distance of running during the whole training program was $8100 \mathrm{~m}$ in specific training sessions and $9600 \mathrm{~m}$ in similar sessions.

The SER50 protocol is presented in Table 5, with a rest period of 2 and 5 minutes between the runs and sets, respec- tively. A training volume progression (of 1 set) was expected, but after four subjects were injured during the first two weeks of training, we decided not to increase the training volume in this training program. The total distance of running during the whole training program was $5400 \mathrm{~m}$ in specific training sessions and $9600 \mathrm{~m}$ in similar sessions.

\section{Measurement of resting hormonal level}

Serum testosterone (TST) and cortisol (COR) were measured for resting hormonal levels (7:00-7:30) at week 1 (before the first training session) and week 6 (before the 18th training session). Also, the testosterone/cortisol ratio (TCR) was calculated and analyzed. Blood was collected into heparinized tubes to obtain plasma. All samples were centrifuged at $2500 \times \mathrm{g}$ for $10 \mathrm{~min}$ at $4{ }^{\circ} \mathrm{C}$, no later than 30 mins after the blood was drawn, and plasma was stored at $-80{ }^{\circ} \mathrm{C}$ for subsequent analyses. All hormones were analyzed by Immunoenzymetric assay ELISA kits (DRG International, Inc., USA; Testosterone EIA-1559 and Cortisol EIA-1887), in accordance with the manufacturer's instructions. Intra-assay variation was 3.34-4.16\% for TST and 3.2-8.1\% for COR. The sensitivity of the assays was $0.083 \mathrm{ng} / \mathrm{ml}$ for TST and $2.5 \mathrm{ng} / \mathrm{ml}$ for COR. All samples of each subject were assayed in duplicate in the same assay to ensure each hormone avoided inter-assay variation. 
Table 4. Characteristics of the SER150 training program

\begin{tabular}{lccccc}
\hline Exercise & Distance & Intensity & $\begin{array}{c}\text { No. of reps } \\
\text { (in 1-3 week) }\end{array}$ & $\begin{array}{c}\text { No. of reps } \\
\text { (in 4-6 week) }\end{array}$ \\
\hline Running & $150 \mathrm{~m}$ & $90-95 \%$ 1RM & 4 & 5 & 1 \\
Running & $150 \mathrm{~m}$ & $90-95 \%$ 1RM & 4 & 5 & 1 \\
Running & $100 \mathrm{~m}$ & $75-80 \%$ 1RM & 8 & 8 & 2 \\
\hline
\end{tabular}

Table 5. Characteristics of the SER50 training program

\begin{tabular}{lccccc}
\hline Exercise & Distance & Intensity & $\begin{array}{c}\text { No. of reps } \\
\text { (in 1-3 week) }\end{array}$ & $\begin{array}{c}\text { No. of reps } \\
\text { (in 4-6 week) }\end{array}$ \\
\hline Running & $50 \mathrm{~m}$ & $95-100 \%$ 1RM & 3 & 3 & 3 \\
Running & $50 \mathrm{~m}$ & $95-100 \%$ 1RM & 3 & 3 & 3 \\
Running & $100 \mathrm{~m}$ & $75-80 \%$ 1RM & 8 & 8 & 2 \\
\hline
\end{tabular}

\section{Physical fitness testing}

The endurance groups (ECR and EIR) were measured by Cooper's 12-minutes run test

This test was performed as described by Bandyopadhyay (2014). All subjects ran on a $350 \mathrm{~m}$ track for a total duration of 12 mins. They were highly motivated to run as many laps as possible and were verbally encouraged to reach their maximal level of effort during the test. The total number of laps was counted and the finishing point marked. Total distance (m) covered in 12 mins was calculated by multiplying the number of complete laps (350 $\mathrm{m}$ ) and the distance covered $(\mathrm{m})$ in the final incomplete lap (Bandyopadhyay, 2014).

The strength groups (RT and ET) were measured by $1 R M$ in the bench press and by power output in the half squat jump

The subjects warmed up as has been described in the "training intervention" section. After a 5 minute rest period, participants performed a lift with load (approx. $80 \%$ of estimated 1RM) through the full range of motion. After each successful performance, the weight was increased (by $2.5-5.0 \mathrm{~kg}$ ) until a failed attempt occurred. The 1RM was achieved in 6-8 attempts. Two min rest periods were given between each attempt in order to reduce fatigue effect. All 1RM measurements were recorded in kilograms for subsequent data analysis.

Following 1RM testing (5 $\mathrm{min}$ ), the power output with the $80 \mathrm{~kg}$ barbell (Pmean80kg) in concentric contraction in the half squat jump was measured with the FiTRODyne premium device (FiTRONiC s. r. o, Bratislava, Slovakia) attached to a barbell and connected to a personal computer. Subjects carried out $2 \times 2$ repetitions with an $80 \mathrm{~kg}$ barbell placed on their shoulders. As described by Vanderka et al. (2016), each half-squat jump set consisted of two repetitions and the squat depth (approx. $90^{\circ}$ knee angle) was controlled by foam cubes, which were $10 \mathrm{~cm}$ in width and individually adjusted for each subject. All subjects squatted down until the hamstrings touched the foam cubes and then jumped as high as possible. During these attempts, the subjects performed the concentric phase of the movement with maximum effort. The attempt with the highest power production was used for further analyses.

The speed-endurance groups (SER50 and SER150) were measured by $50 \mathrm{~m}$ and $150 \mathrm{~m}$ sprint tests

Firstly, the subjects' 50 m run times were measured according to the methods of Vanderka et al. (2016). Dual-beam light timing gates (Vanel-gates, Vanel Ltd, Nizna, Slovakia) were used.
Subjects began from a stationary, standing start; the front foot was placed $50 \mathrm{~cm}$ behind the first timing gate (i.e. $-0.5 \mathrm{~m}$ ) to avoid spontaneous triggering of the timing gates (i.e. arm wave). All subjects performed two trials and ran as fast as possible throughout the entire $50 \mathrm{~m}$ distance. The rest interval between trials was 5 minutes. Subsequently, 5 mins after the last $50 \mathrm{~m}$ run, a second running test was carried out under similar conditions. Subjects performed $2 \times 150$ m maximum sprints with a 2 min rest period. The short rest period was chosen due to the potential for analysis of fatigue index. During the $150 \mathrm{~m}$ sprint test, the time for $100 \mathrm{~m}$ was registered to establish training intensity in a similar session. The best times in the 50 and $150 \mathrm{~m}$ sprints were recorded for further analysis. An indoor $(200 \mathrm{~m})$ track was chosen to create standardized conditions for the sprint test.

\section{Statistical analyses}

Standard methods were used to determine means and standard deviations. Statistical assumptions for each variable were performed using the Shapiro-Wilk test and Levene's test. For hormonal analyses, the main effects of the training were analyzed by repeated-measures ANOVA (six groups $\times$ two testing points). Bonferroni post hoc tests were applied to determine the source of differences. For physical fitness tests, the dependent samples $T$-test was used to establish changes within the groups. Subsequently, differences between groups (specific for endurance, strength and speed-endurance) in absolute changes $(\Delta)$ over time were assessed by independent $T$-test. Cohen's $d$ was used and interpreted as $<0.2$ is a small effect, $0.2-0.8$ is a moderate effect, and $>0.8$ is a large effect. $95 \%$ confidence intervals $(95 \% \mathrm{CI}$ ) were also calculated. Statistical significance was accepted at $p \leq 0.05$. Each statistical analysis was carried out by SPSS 23 statistical software (IBM, New York, USA).

\section{Results}

\section{Resting hormonal level}

The main effects of the training on resting hormonal level were observed (COR, $F=4.147, p=0.045$ and $\mathrm{T} / \mathrm{CR}, F=8.154$, $p=0.006$ ) in pre (week 1 ) to post (week 6) comparison. There were differences between the groups in relation to changes of resting level COR (ECR vs. ET, $p=0.036$; ECR vs. SER50, $p=0.001$ ); (EIR vs. ET, $p=0.014$; EIR vs. SER50, $p=0.001$ ) 
and (RT vs. ET, $p=0.015$; RT vs. SER50, $p=0.001$ ). For T/CR, differences between the groups were observed (ECR vs. ET, $p=0.002$; ECR vs. SER50, $p=0.001$ and ECR vs. SER150, $p=0.001$ ) and (RT vs. SER50, $p=0.042$ and RT vs. SER150, $p=0.027$ ), respectively. A summary of the results of resting hormonal level of each group is presented in Table 6 .

After 6 weeks of training, the resting level of COR decreased in ECR $(t=-2.737, p=0.016,95 \% C I=-60.45$ to $-7.33 \mathrm{ng} / \mathrm{ml})$, EIR $(t=-2.528, p=0.027,95 \% \mathrm{CI}=-50.91$ to
$-3.77 \mathrm{ng} / \mathrm{ml})$ and RT $(t=-2.199, p=0.045,95 \% \mathrm{CI}=-48.80$ to $-0.61 \mathrm{ng} / \mathrm{ml}$ ) compared to pre-values. Otherwise an increase of COR $(t=4.142, p=0.006,95 \% C I=10.89$ to $42.33 \mathrm{ng} / \mathrm{ml})$ was observed in the SER50 group. T/C ratio significantly elevated in ECR ( $t=3.107, p=0.008,95 \% C I=3.96$ to 21.66$)$ and $\operatorname{EIR}(t=2.816, p=0.016,95 \% \mathrm{CI}=1.37$ to 10.77$)$ and decreased in the SER50 group $(t=-3.263, p=0.017,95 \% C I=-8.38$ to -1.19). There were no statistically significant differences in the resting level of TST compared to pre values in any groups.

Table 6. Resting hormonal level in pre- and post-training in each training group (mean \pm SD)

\begin{tabular}{|c|c|c|c|c|}
\hline Group & Hormones & Pre & Post & Cohen's d \\
\hline \multirow{3}{*}{$\mathrm{ECR}$} & Cortisol (ng/ml) & $214.91 \pm 42.72$ & $181.03 \pm 57.90^{*}$ & 0.50 \\
\hline & Testosterone (ng/ml) & $5.97 \pm 1.61$ & $6.99 \pm 2.05$ & 0.35 \\
\hline & $\mathrm{T} / \mathrm{C}$ ratio & $29.00 \pm 10.36$ & $41.82 \pm 16.46^{*}$ & 0.57 \\
\hline \multirow{3}{*}{ EIR } & Cortisol (ng/ml) & $205.55 \pm 41.91$ & $178.20 \pm 22.10^{*}$ & 0.50 \\
\hline & Testosterone (ng/ml) & $5.27 \pm 1.48$ & $5.66 \pm 1.50$ & 0.28 \\
\hline & $\mathrm{T} / \mathrm{C}$ ratio & $26.26 \pm 7.83$ & $32.33 \pm 10.24^{*}$ & 0.55 \\
\hline \multirow{3}{*}{ RT } & Cortisol (ng/ml) & $206.34 \pm 45.51$ & $181.63 \pm 51.06^{*}$ & 0.40 \\
\hline & Testosterone (ng/ml) & $5.75 \pm 1.58$ & $5.63 \pm 1.06$ & 0.07 \\
\hline & $\mathrm{T} / \mathrm{C}$ ratio & $29.05 \pm 9.72$ & $33.17 \pm 10.59$ & 0.30 \\
\hline \multirow{3}{*}{ ET } & Cortisol (ng/ml) & $247.78 \pm 47.17$ & $235.70 \pm 43.73$ & 0.21 \\
\hline & Testosterone (ng/ml) & $5.780 \pm 1.65$ & $5.672 \pm 1.36$ & 0.04 \\
\hline & $\mathrm{T} / \mathrm{C}$ ratio & $23.64 \pm 6.15$ & $24.79 \pm 6.03$ & 0.13 \\
\hline \multirow{3}{*}{ SER50 } & Cortisol (ng/ml) & $261.68 \pm 52.90$ & $288.30 \pm 46.29^{*}$ & 1.11 \\
\hline & Testosterone (ng/ml) & $5.77 \pm 1.03$ & $5.29 \pm 1.31$ & 0.21 \\
\hline & $\mathrm{T} / \mathrm{C}$ ratio & $22.32 \pm 4.52$ & $17.53 \pm 4.33^{*}$ & 0.82 \\
\hline \multirow{3}{*}{ SER150 } & Cortisol (ng/ml) & $231.33 \pm 39.70$ & $241.62 \pm 29.64$ & 0.16 \\
\hline & Testosterone (ng/ml) & $4.63 \pm 1.21$ & $5.01 \pm 0.71$ & 0.29 \\
\hline & $\mathrm{T} / \mathrm{C}$ ratio & $20.80 \pm 7.54$ & $21.07 \pm 4.13$ & 0.03 \\
\hline
\end{tabular}

* significant difference from pre values at $p \leq 0.05$. T/C ratio - Testosterone/Cortisol ratio.

\section{Endurance training groups}

Both groups improved the distance covered during Cooper's 12 -minute run test from pre- to post-training ECR $(t=3.502$, $p=0.004,95 \% C I=48.30$ to $201.30 \mathrm{~m})$ and $\operatorname{EIR}(t=3.144$, $p=0.008,95 \% C I=34.48$ to $190.13 \mathrm{~m}$ ), respectively. There were no differences between the groups at either time point or in the change from week 1 to week 6.

\section{Strength training groups}

The 1RM increased from pre- to post-training in both RT groups $(t=2.781, p=0.016,95 \% \mathrm{CI}=1.01$ to $8.05 \mathrm{~kg}$ ) and ET groups $(t=3.791, p=0.002,95 \% \mathrm{CI}=1.97$ to $6.97 \mathrm{~kg}$ ). Similar results were observed in Pmean80kg. In both groups, RT ( $t=3.111, p=0.008,95 \% \mathrm{CI}=23.26$ to $126.57 \mathrm{w})$ and $\mathrm{ET}$ $(t=4.100, p=0.001,95 \% C I=43.69$ to $137.24 \mathrm{w})$ power output increase significantly. There were no differences between the groups at either time point or in the change from week 1 to week 6 in both 1RM and Pmean80kg tests.

\section{Speed-endurance groups}

Compared to pre-training, the SER150 group showed a significantly decreased time $(t=-3.624, p=0.005,95 \% C I=-0.70$ to $-0.17 \mathrm{~s}$ ) for the $150 \mathrm{~m}$ sprint test, while the results of the SER50 group remained unchanged in both tests. The SER150 group also showed positive trends for the $50 \mathrm{~m}$ sprint test $(t=-2.061, p=0.066,95 \% C I=-0.21$ to $-0.01 \mathrm{~s})$. No differences were registered in pre-training (week 1 ) values. However, significant differences between the groups $(t=2.259$, $p=0.037$ ) were found in changes from pre- to post-training in the $150 \mathrm{~m}$ test.

\section{Discussion}

The primary purpose of this study was to determine the response of the resting level of serum cortisol (COR), testosterone (TST) and testosterone/cortisol ratio (T/C) to different training modalities in physically active young males. A secondary purpose was to identify if the different training programs are an effective way to improve physical fitness according to the type of training modalities performed during six-week training programs.

To the best of our knowledge, this is the first study that compares the effects of three different training modalities (endurance, strength, and speed-endurance) in combination with varying intensities on the resting hormonal level and physical fitness within a short-term duration among active young males. Our results demonstrate that the endurance (both ECR and EIR) and the strength (RT) training programs were modalities that positively affect the endocrine system via reduction of the resting level of COR. Also, both ECR and EIR led to increased T/C marker. On the contrary, SER50 was the only training program that led to a significant increase of COR and decreased T/C ratio marker. Moreover, except for the SER50 training programs, each training program led to a significant improvement in physical fitness - as measured by specific tests 
Table 7. Physical performance in pre- and post-training in each training group (mean \pm SD)

\begin{tabular}{|c|c|c|c|c|}
\hline Group & Test & Pre & Post & Cohen's d \\
\hline ECR & Cooper's 12-minute run test (m) & $2999 \pm 297$ & $3124 \pm 223^{*}$ & 0.64 \\
\hline EIR & Cooper's 12-minute run test $(\mathrm{m})$ & $2913 \pm 251$ & $3026 \pm 156^{*}$ & 0.62 \\
\hline RT & $\begin{array}{c}\text { 1RM (kg) } \\
\text { Pmean80kg (w) }\end{array}$ & $\begin{array}{c}89 \pm 13 \\
1535 \pm 140\end{array}$ & $\begin{array}{c}94 \pm 14^{*} \\
1609 \pm 189^{*}\end{array}$ & $\begin{array}{l}0.53 \\
0.58\end{array}$ \\
\hline ET & $\begin{array}{c}\text { 1RM (kg) } \\
\text { Pmean80kg (w) }\end{array}$ & $\begin{array}{c}92 \pm 9 \\
1515 \pm 142\end{array}$ & $\begin{array}{c}96 \pm 11^{*} \\
1606 \pm 125^{*}\end{array}$ & $\begin{array}{l}0.65 \\
0.70\end{array}$ \\
\hline SER50 & $\begin{array}{l}50 \mathrm{~m} \text { sprint test }(\mathrm{s}) \\
150 \mathrm{~m} \text { sprint test }(\mathrm{s})\end{array}$ & $\begin{array}{c}6.58 \pm 0.27 \\
19.75 \pm 0.98\end{array}$ & $\begin{array}{c}6.61 \pm 0.24 \\
19.71 \pm 0.80\end{array}$ & $\begin{array}{l}0.18 \\
0.11\end{array}$ \\
\hline SER150 & $\begin{array}{l}50 \mathrm{~m} \text { sprint test (s) } \\
150 \mathrm{~m} \text { sprint test }(\mathrm{s})\end{array}$ & $\begin{array}{c}6.60 \pm 0.30 \\
19.79 \pm 1.09\end{array}$ & $\begin{array}{c}6.49 \pm 0.30 \\
19.36 \pm 1.08^{*}\end{array}$ & $\begin{array}{l}0.44 \\
0.77\end{array}$ \\
\hline
\end{tabular}

${ }^{*}$ significant difference from pre values at $p \leq 0.05 .1 \mathrm{RM}$ - One repetition maximum in bench press; Pmean80kg - power output with $80 \mathrm{~kg}$ barbell in the half-squat jump.

according to the type of training modalities performed in each program.

As was mentioned in the introduction, changes of hormonal level depend on the characteristics of the samples (gender, age, training experiences, etc.) as well as training program variables (duration of the training program, frequency of training, modality, intensity, volume, etc.). In accordance with this knowledge, the results of this study were compared to other studies with a similar sample of participants' and training program variables.

TST is the primary anabolic hormone, and its biological effects include the stimulation of protein synthesis (anabolic effect) and inhibiting the protein degradation anti-catabolic effect (Vingren et al., 2010). A physiological level of TST is necessary for regular strength and muscle adaptation to training (Kvorning et al., 2006). Firstly, all subjects were in the physiological range for resting level of TST. In the present study, no changes were observed in the resting level of TST following any of the training programs., The role of TST changes induced by exercise training and the adaptation to the training program has not been not well explored yet and contraindicative results exist in the literature (Ahtiainen et al., 2003; Alghadir et al., 2015; Grandys et al., 2009; Kraemer et al., 1999; Shakeri et al., 2012). A current review by Hooper et al. (2017) suggests that neither an acute rise in TST following resistance exercise nor a long-term basal increase in TST is necessary to experience gains in strength and hypertrophy. This suggestion is in line with our results because both strength training programs (RT and ET) showed a significant increase in performance (1RM and Pmean80kg) without changes in the resting level of TST.

Moreover, similar results were observed in the endurance (ECR, EIR) and speed-endurance (SER150) training programs. Our results suggest that improvement in physical fitness of different training modalities can be reached without changes in the resting level of TST in active males. Also, we recommend that resting testosterone is not an appropriate parameter of positive or negative adaptation to physical training in subjects within a normal physiological range during a short-term training program.

One of the main findings of the present study was that all groups have a high level of resting COR during the pre-testing. The upper limit in the range of the reference values for the national population is $224 \mathrm{ng} / \mathrm{ml}$. In the present study, three groups were close to the limit $(\mathrm{ECR}=214.91 \pm 42.72$; $\mathrm{EIR}=205.55 \pm 41.91 ; \mathrm{RT}=206.34 \pm 45.51 \mathrm{ng} / \mathrm{ml})$ and anoth- er three were over $(\mathrm{ET}=247.78 \pm 47.17$; SER50 $=261.68 \pm$ 52.90, SER150 $=231.33 \pm 39.70 \mathrm{ng} / \mathrm{ml}$ ) at pre-training testing. A possible explanation of these high values is, as has been published before (Sedliak et al., 2007), that high level of COR can be considered as a result of higher anticipatory stress before the initial testing and that anticipatory COR responses had already disappeared after the first testing (Mason et al., 1973). It should be noted that this phenomenon could affect our results.

COR induces catabolic effects in different tissues, such as skeletal muscle, bone, and heart muscle, among others. Increase of resting level of COR can negatively affect the adaptation of these tissues to training via inhibition of protein synthesis and muscle growth (Hackney and Walz, 2013). Chwalbińska-Moneta et al. (2005) showed that moderate endurance training (similar to ECR) in young males induces a decrease in COR already after the first week of training, which is considered as an early hormonal effect of physical activity. A further reduction in the resting level of COR was observed within the following two weeks of training, which may partially indicate an exercise adaptation to the training program. Also, Shakeri et al. (2012) observed a significant reduction of resting COR and increased $\mathrm{T} / \mathrm{C}$ ratio, while $\mathrm{TST}$ remained unchanged within the following six weeks of the moderate-intensity endurance training program. Our results are in line with Shakeri et al. (2012), but a similar response of resting COR and TST was observed in both ECR and EIR as well as in the RT program. In these training programs, a moderate to vigorous intensity was involved. The reduction of resting COR levels observed in the present study can be beneficial for both health and performance status, as it should allow the desired adaptation of whole structures of the body to training. The decrease of COR could also have had a positive effect on the improvement in physical fitness.

$\mathrm{T} / \mathrm{C}$ ratio is a variable currently used in the evaluation of the organism response to acute and chronic physical stressors (De Luccia, 2016). Earlier, the relationship between cortisol and testosterone, reflecting anabolic-catabolic balance and the $\mathrm{T} / \mathrm{C}$ ratio was considered as a marker of overtraining syndrome (Adlercreutz et al., 1986). The results of Fry and Kraemer (1997) supported this suggestion; they found that the high volume and/or high intensity related to overreaching or overtraining decreasing $\mathrm{T} / \mathrm{C}$ ratio by increases resting level of COR and decreases resting TST levels. The results of Fry and Kraemer (1997) supported this suggestion; they found that 
the high volume and/or high intensity related to overreaching or overtraining negatively affect $\mathrm{T} / \mathrm{C}$ ratio by increases resting level of COR and decreases resting TST levels.

Thus, it seemed that the combination of volume and intensity of the training programs used in the present study were not associated with overreaching and/or overtraining symptoms. However, the current findings of a systematic review of Cadegiani and Kater (2018) conclude that the COR and T/C ratio are not a good predictor of overtraining syndrome. The most current consensus indicated that the $\mathrm{T} / \mathrm{C}$ ratio is related to the duration and intensity of training, and this ratio indicates only the tangible physiological strain of training (De Luccia, 2016). In the present study, a significant increase of the $\mathrm{T} / \mathrm{C}$ ratio was found in those following the ECR and EIR training program, while no changes were observed in other training programs. These changes have been mainly caused by a significant reduction of resting level of cortisol, while testosterone level remains unchanged. These results suggest that ECR and EIR training programs (which involved moderate to vigorous intensity, total training session duration 30-60 minutes, performed three times per week) present a suitable combination of intensity and duration of training sessions which lead to a positive hormonal balance between resting COR and TST level.

The evidence suggests that overloading training can lead to the chronic catabolic state - which can be reflected by the increase of resting COR level, and thus, may negatively affect adaptation to training (Cadore et al., 2012). This mechanism could affect the response of the SER50 group to the training program. SER50 is the only group in which the resting level of COR and $\mathrm{T} / \mathrm{C}$ ratio negatively changed while physical performance remained unchanged. Additionally, in the first three weeks, four subjects were injured during this training program (hamstring injuries), and so volume progression was not applied. Taken together, SER50 (high-intensity interval training, which incorporated running as a training activity) performed with maximum intensity had a negative effect on the hormonal response of resting COR as well as the incidence of injuries. Based on our results, SER50 protocol is not appropriate for active males without sprint training backgrounds, and the practical application of this type of training program has to be deeply considered in both research and training.

From a practical perspective, each training program has had a moderate improvement effect on physical performance (in the range of $d=0.44-0.77$ ) - except for the SER50 training program. Thus, it seems that the improvement of physical fitness is independent of changes in resting hormonal level mediated by training programs. Based on the data, it appears that a 6-week training period seems to be long enough to significantly improve strength, endurance, and speed-endurance in previously active males if the intensity and volume are appropriate.

Due to its relevance for both physical fitness and health, further research is recommended with other highly used training programs (such as high-intensity interval training, eccentric strength training or training with vascular occlusion) and a large sample size with both genders and/or individuals with some form of physical or medical impairment.

\section{Conclusions}

These results suggest that the effects of the training program on resting hormonal concentration are modified more by the intensity than the modalities of training programs. The ECR, EIR and RT training programs were performed with a moder- ate to vigorous intensity, which seems to be a key factor that affects hormonal responses to training programs. The training programs used in this study were designed to be as similar as possible to those used in practice by active people in their physical activity programs or among recreationally trained athletes. According to our results, the endurance and resistance training modalities performed with a moderate to vigorous intensity may be a usable way to manage the resting cortisol level and enhance physical fitness in active young males.

\section{Conflict of interests}

The authors have no conflict of interests to declare.

\section{Funding and acknowledgements}

This study was supported by The Ministry of Education, Science, Research and Sport of the Slovak Republic, grant VEGA number 1/0291/14 and 1/0608/20.

\section{References}

Adlercreutz H, Härkönen M, Kuoppasalmi K, Näveri H, Huhtaniemi I, Tikkanen H, et al. (1986). Effect of training on plasma anabolic and catabolic steroid hormones and their response during physical exercise. Int J Sports Med 7(Suppl. 1): 27-28. DOI: 10.1055/s-2008-1025798.

Ahtiainen JP, Pakarinen A, Alen M, Kraemer WJ, Häkkinen K (2003). Muscle hypertrophy, hormonal adaptations and strength development during strength training in strength-trained and untrained men. Eur J Appl Physiol 89(6): 555-563. DOI: 10.1007/ s00421-003-0833-3.

Alghadir AH, Gabr SA, Aly FA (2015). The effects of four weeks aerobic training on saliva cortisol and testosterone in young healthy persons. J Phys Ther Sci 27(7): 2029-2033. DOI: 10.1589/ jpts.27.2029.

Bandyopadhyay A (2014). Validity of Cooper's 12-min run test for estimation of maximum oxygen uptake in female university students. Indian J Physiol Pharmacol 58(2): 184-186. DOI: $10.5604 / 20831862.1127283$.

Cadegiani FA, Kater CE (2018). Hormonal response to a nonexercise stress test in athletes with overtraining syndrome: results from the Endocrine and metabolic Responses on Overtraining Syndrome (EROS) - EROS-STRESS. J Sci Med Sport 21(7): 648-653. DOI: 10.1016/j.jsams.2017.10.033.

Cadore EL, Izquierdo M, dos SantosMG, Martins JB, Rodrigues Lhullier FL, Pinto RS, et al. (2012). Hormonal responses to concurrent strength and endurance training with different exercise orders. J Strength 26(12): 3281-3288. DOI: 10.1519/ JSC.0b013e318248ab26.

Caspersen CJ, Powell KE, Christenson GM (1985). Physical activity, exercise, and physical fitness: definitions and distinctions for health-related research. Public Health Rep 100(2): 126-131.

Chwalbińska-Moneta J, Kruk B, Nazar K, Krzemiński K, KaciubaUściłko H, Ziemba A (2005). Early effects of short-term endurance training on hormonal responses to graded exercise. J Physiol Pharmacol 56(1): 87-99.

De Luccia TPB (2016). Use of the testosterone/cortisol ratio variable in sports. Open Sports Sci J 9: 104-113. DOI: 10.2174/1875399X01609010104.

Doma K, Schumann M, Sinclair WH, Leicht AS, Deakin GB, Häkkinen K (2015). The repeated bout effect of typical lower body strength training sessions on sub-maximal running performance and hormonal response. Eur J Appl Physiol 115(8): 1789-1799. DOI: 10.1007/s00421-015-3159-z.

Fry AC, Kraemer WJ (1997). Resistance exercise overtraining and overreaching. Neuroendocrine responses. Sports Med 23(2): 106-129. DOI: 10.2165/00007256-199723020-00004.

Grandys M, Majerczak J, Duda K, Zapart-Bukowska J, Kulpa J, Zoladz JA (2009). Endurance training of moderate intensity 
increases testosterone concentration in young, healthy men. Int J Sports Med 30(7): 489-495. DOI: 10.1055/s-0029-1202340.

Hackney AC, Walz EA (2013). Hormonal adaptation and the stress of exercise training: the role of glucocorticoids. Trends Sport Sci 20(4): 165-171.

Häkkinen K, Alen M, Kallinen M, Newton RU, Kraemer WJ (2000). Neuromuscular adaptation during prolonged strength training, detraining and re-strength-training in middle-aged and elderly people. Eur J Appl Physiol 83(1): 51-62. DOI: 10.1007/ s004210000248.

Häkkinen K, Alen M, Kraemer WJ, Gorostiaga E, Izquierdo M, Rusko H, et al. (2003). Neuromuscular adaptations during concurrent strength and endurance training versus strength training. Eur J Appl Physiol 89(1): 42-52. DOI: 10.1007/s00421002-0751-9.

Hawley JA (2008). Specificity of training adaptation: time for a rethink? J Physiol 586(1): 1-2. DOI: 10.1113/ jphysiol.2007.147397.

Hooper DR, Kraemer WJ, Focht BC, Volek JS, DuPont WH, Caldwell LK, Maresh CM (2017). Endocrinological Roles for Testosterone in Resistance Exercise Responses and Adaptations. Sports Med 47(9): 1709-1720. DOI: 10.1007/s40279-017-0698-y.

Hough J, Corney R, Kouris A, Gleeson M (2013). Salivary cortisol and testosterone responses to high-intensity cycling before and after an 11-day intensified training period. J Sports Sci 31(14): 1614-1623. DOI: 10.1080/02640414.2013.792952.

Kilian Y, Engel F, Wahl P, Achtzehn S, Sperlich B, Mester J (2016). Markers of biological stress in response to a single session of high-intensity interval training and high-volume training in young athletes. Eur J Appl Physiol 116(11-12): 2177-2186. DOI: 10.1007/s00421-016-3467-y.

Kraemer WJ, Fleck SJ, Evans WJ (1996). Strength and power training: physiological mechanisms of adaptation. Exerc Sport Sci Rev 24: 363-397.

Kraemer WJ, Häkkinen K, Newton RU, Nindl BC, Volek JS, McCormick M, et al. (1999). Effects of heavy-resistance training on hormonal response patterns in younger vs. older men. J Appl Physiol 87(3): 982-992. DOI: 10.1152/jappl.1999.87.3.982.

Kvorning T, Andersen M, Brixen K, Madsen K (2006). Suppression of endogenous testosterone production attenuates the response to strength training: a randomized, placebo-controlled, and blinded intervention study. Am J Physiol Endocrinol Metab 291(6): E1325-E1332. DOI: 10.1152/ajpendo.00143.2006.
Mason JW, Hartley LH, Kotchen TA, Mougey EH, Ricketts PT, Jones LG (1973). Plasma cortisol and norepinephrine responses in anticipation of muscular exercise. Psychosom Med 35(5): 406-414. DOI: 10.1097/00006842-197309000-00004.

Peake JM, Tan SJ, Markworth JF, Broadbent JA, Skinner TL, Cameron-Smith D (2014). Metabolic and hormonal responses to isoenergetic high-intensity interval exercise and continuous moderate-intensity exercise. Am J Physiol Endocrinol Metab 307(7): E539-E552. DOI: 10.1152/ajpendo.00276.2014.

Popovic B, Popovic D, Macut D, Antic IB, Isailovic T, Ognjanovic S, et al. (2019). Acute Response to Endurance Exercise Stress: Focus on Catabolic/anabolic Interplay Between Cortisol, Testosterone, and Sex Hormone Binding Globulin in Professional Athletes. J Med Biochem 38(1): 6-12. DOI: 10.2478/jomb-2018-0016.

Schumann M, Mykkänen OP, Doma K, Mazzolari R, Nyman K, Häkkinen K (2015). Effects of endurance training only versus same-session combined endurance and strength training on physical performance and serum hormone concentrations in recreational endurance runners. Appl Physiol Nutr Metab 40(1): 28-36. DOI: 10.1139/apnm-2014-0262.

Sedliak M, Finni T, Cheng S, Kraemer WJ, Häkkinen K (2007). Effect of time-of-day-specific strength training on serum hormone concentrations and isometric strength in men. Chronobiol Int 24(6): 1159-1177. DOI: 10.1080/07420520701800686.

Shakeri N, Nikbakht H, Azarbayjani M, Amirtash AM (2012). The effect of different types of exercise on the testosterone/cortisol ratio in untrained young males. Ann Biol Res 3(3):1452-1460.

Vanderka M, Longová K, Olasz D, Krčmár M, Walker S (2016). Improved Maximum Strength, Vertical Jump and Sprint Performance after 8 Weeks of Jump Squat Training with Individualized Loads. J Sports Sci Med 15(3): 492-500.

Vingren JL, Kraemer WJ, Ratamess NA, Anderson JM, Volek JS, Maresh CM (2010). Testosterone physiology in resistance exercise and training: the up-stream regulatory elements. Sports Med 40(12): 1037-1053. DOI: 10.2165/11536910-000000000-00000.

Walker S, Häkkinen K, Haff GG, Blazevich AJ, Newton RU (2017). Acute elevations in serum hormones are attenuated after chronic training with traditional isoinertial but not accentuated eccentric loads in strength-trained men. Physiol Rep 5(7): e13241. DOI: 10.14814/phy2.13241. 